\section{Établissement du microbiote}

\section{Une colonisation in utero déterminante pour la santé future?}

> Certaines pathologies semblent avoir une origine développementale et, aujourd'hui, le microbiote apparaît comme un déterminant de l'état de santé de l'homme et son établissement, une étape importante chez le nouveau-né. Des variations dans sa constitution, incluant un déséquilibre (ou dysbiose), ont ainsi été associées à de nombreuses pathologies. De récents travaux suggèrent qu'une colonisation bactérienne de l'utérus, du liquide amniotique ou encore du placenta, des sites auparavant pensés stériles, existerait. Durant les phases de son développement, le fœtus pourrait ainsi rencontrer des bactéries in utero. Elles contribueraient à l'établissement de son microbiote avant même l'accouchement et donc avant la rencontre avec les microorganismes des microbiotes vaginal, fécal et cutané, ceux-ci variant selon les modes d'accouchement (voie basse ou césarienne). Les premières études sur l'existence d'un microbiote in utero, qui se caractérise par une faible biomasse, sont cependant controversées.

\section{L’origine développementale des maladies}

II y a presque 40 ans, une théorie selon laquelle l'origine de certaines pathologies ne résulterait pas uniquement de l'environnement qui serait associé à un bagage génétique défavorable, mais également d'événements survenus durant les phases de développement de l'individu, a été proposée. C'est le concept de l'origine développementale de la santé et des maladies, connue par son acronyme anglais DOHaD (developmental ori-

$(\rightarrow)$ Voir le numéro thématique $\mathrm{DOHaD}$, gin of health and diseases) $(\rightarrow)$. pouvant être héréditaires, de l'expression de gènes sans qu'il n'y aient de changements de séquence nucléoti-

Vignette (Photo $\odot$ Sandra Wydau-Dematteis) $\mathrm{m} / \mathrm{s} \mathrm{n}^{\circ} 1$, janvier 2016

L'épigénétique, qui correspond à des modifications,

\section{Rémi Gschwind ${ }^{1}$, Thierry Fournier ${ }^{2}$, Marie-José Butel ${ }^{1}$, Sandra Wydau-Dematteis ${ }^{1}$}

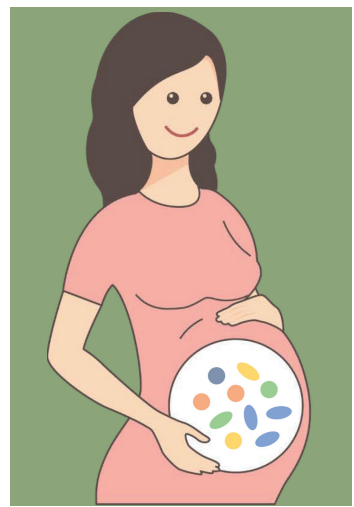

${ }^{1} \varepsilon A 4065$, Faculté de pharmacie de Paris, DHU Risques et grossesse, université Paris Descartes, 4, avenue de l'Observatoire, 75006 Paris, France.

${ }^{2}$ UMR-S1139, Inserm, Faculté de pharmacie de Paris, DHU Risques et grossesse, fondation PremUp, université Paris Descartes,

4, avenue de l'observatoire, 75006 Paris, France.

sandra.wydau@parisdescartes.fr

dique de l'ADN, est un élément important de ce concept $[1](\rightarrow)$.

En effet, des marques épigénétiques (comme la

$(\rightarrow)$ Voir la Synthèse de M.A. Charles et al., $\mathrm{m} / \mathrm{s} \mathrm{n}^{\circ} 1$, janvier 2016, page 15 méthylation de l'ADN, des modifications au niveau des histones ou encore l'expression d'ARN non codants) influent sur le niveau d'expression de certains gènes avec, pour conséquence, des modifications qui touchent des lignées cellulaires, des voies métaboliques ou encore des organes entiers, selon le stade de développement auquel elles sont apparues [1, 2]. Ces phases du développement constituent donc une fenêtre de plasticité pour la mise en place d'un capital fonctionnel et métabolique. Un environnement défavorable subi lors de la grossesse ou quelques temps après la naissance peut ainsi modifier de manière permanente la structure, la fonction ou le métabolisme de certains organes. Chez l'enfant de moins de 2 ans, la malnutrition ou le manque d'affection peuvent ainsi laisser des marques épigénétiques sur l'ADN. L'obésité maternelle, le diabète gestationnel ou l'exposition à des substances toxiques (xénobiotiques, tabac, pollution, perturbateurs endocriniens [2]) durant la grossesse, peuvent également laisser des marques épigénétiques sur l'ADN du futur enfant. Ces marques entraîneront, par la suite, une sensibilité accrue à un événement défavorable pouvant provoquer certaines maladies (hypertension, maladie cardiovasculaire, hyperactivité, trouble autistique) [1]. Ces différentes observations ont conduit à définir une période critique de 1000 jours s'étalant de la conception de l'enfant à ses 2 ans [3] $(\rightarrow)$.

$(\rightarrow)$ Voir l’éditorial de M.N. Bruné Drisse, $m / s$ $n^{\circ} 1$, janvier 2016, page 9 De nombreuses études épidémiologiques chez l'homme et expérimentales chez l'animal ont mis en évidence cette contribution des phases de développement dans l'apparition des maladies. Pendant 
la grossesse, ces stress environnementaux sont tamponnés par le placenta qui, grâce à sa plasticité, s'adapte à son environnement pour promouvoir la santé du fœtus et la poursuite de la grossesse. Le placenta est un organe primordial et spécifique de la grossesse, qui permet les échanges entre la mère et le fœtus. II protège également le fœtus par son rôle de barrière contre les pathogènes et certains xénobiotiques ou substances toxiques. L'organe de la grossesse se retrouve ainsi au cœur de cette programmation de l'état de santé futur [4].

\section{Le microbiote, partenaire de la santé}

Aujourd'hui, l'importance du microbiote, c'est-à-dire l'ensemble des communautés bactériennes vivant en symbiose avec l'organisme qui les héberge, est reconnue dans la communauté scientifique. Le corps abrite environ le même nombre de cellules eucaryotes que de cellules procaryotes [5]. Dans certaines niches, comme par exemple l'intestin, le microbiote est désormais considéré comme un organe à part entière en raison des nombreuses fonctions qui lui sont attribuées. En effet, le microbiote exerce une activité de barrière contre les microorganismes pathogènes, en mettant en place une compétition pour les divers nutriments dont les bactéries ont besoin pour se multiplier, mais également en produisant certains métabolites antimicrobiens. Le microbiote participe aussi à la maturation et à l'homéostasie du système immunitaire. Grâce à des boucles de rétrocontrôles complexes, il établit un dialogue avec le système immunitaire de l'hôte permettant de maintenir une réponse efficace contre les pathogènes et d'induire une tolérance vis-à-vis de certains antigènes considérés comme inoffensifs. Il exerce enfin de nombreuses fonctions métaboliques: aide à la digestion de certains nutriments, production de métabolites essentiels et de vitamines. De nombreuses études ont démontré que des déséquilibres du microbiote, ou dysbioses [6], étaient associés à diverses pathologies, comme les maladies inflammatoires chroniques de l'intestin ( $\mathrm{MICl})$, l'obésité ou des maladies comme l'autisme ou l'état dépressif [7]. Par ses activités bénéfiques, le microbiote fécal est désormais considéré comme un médicament par la législation française $(\rightarrow)$. Des transferts de microbiote fécal sont ainsi prescrits dans le cadre d'infections

$(\rightarrow)$ Voir le numéro thématique Le microbiote, $\mathrm{m} / \mathrm{s} \mathrm{n}^{\circ} 11$, novembre 2016 récurrentes à Clostridium difficile [8], et des essais cliniques sont réalisés pour d'autres pathologies ( $\mathrm{MICl}$, maladies métaboliques, syndrome de l'intestin irritable, infections par des pathogènes multi-résistants [9]).

\section{L'établissement du microbiote, un rôle dans l'état de santé à long terme?}

Les importantes fonctions physiologiques du microbiote intestinal ainsi que les relations établies entre dysbiose et certaines pathologies ont conduit à s'intéresser à son établissement dans les premières semaines de vie, une période reconnue aujourd'hui comme une étape clé [10]. La colonisation bactérienne du fœtus débute au moment de la rupture des membranes, peu de temps avant la naissance. Elle est suivie d'une colonisation massive lors de l'accouchement majoritairement par l'inhalation et/ou l'ingestion des microbiotes vaginal, fécal et cutané de la mère, selon les modes d'accouchement. De plus en plus d'importance est donnée à ces premières bactéries colonisatrices qui sont supposées influer sur la santé future de la mère et de l'enfant. Les bactéries peuvent induire certaines modifications épigénétiques, un élément important dans le concept de la DOHaD [11], et la mise en place de nombreuses fonctions physiologiques, en particulier les fonctions de barrière et de maturation immunitaire.

De nombreuses études ont relié des facteurs responsables de modifications précoces du microbiote intestinal du nouveau-né avec la survenue ultérieure de pathologies, permettant d'inclure l'établissement du microbiote dans le concept de la DOHaD. Des associations entre la naissance par césarienne ou une antibiothérapie néonatale, facteurs reconnus pour altérer significativement l'établissement du microbiote, et l'augmentation du risque de développer des pathologies telles que l'eczéma, l'allergie, l'asthme ou une MICI ont ainsi été observées [12]. Au contraire, un contact avec une grande diversité de microorganismes, comme c'est le cas dans un environnement rural ou en présence d'animaux de compagnie, apparaît être protecteur visà-vis de ces pathologies [13].

Auparavant, le milieu in utero était considéré stérile. Plusieurs études ont récemment montré que le méconium, première selle émise par le nouveau-né, reflétant donc son environnement in utero, n'était pas stérile [14]. L'existence d'un microbiote in utero constituerait donc le premier inoculum bactérien qui pourrait influer sur la physiologie du fœtus immature. Une réelle homéostasie est présente au niveau du placenta. Ainsi, parmi d'autres facteurs, l'immunité maternelle, l'immunité fœtale et les bactéries commensales présentes à ce niveau participent au maintien de cette homéostasie. Cet équilibre, qui permet d'assurer le bon déroulement de la grossesse et d'éviter les infections tout en tolérant les bactéries commensales, pourrait être important pour la grossesse mais également pour la maturation du système immunitaire et l'état de santé futur de l'enfant. Le microbiote in utero rejoindrait alors le concept de la $\mathrm{DOHaD}$ et constituerait un paramètre important à considérer dans la théorie des 1000 jours (Figure 1).

\section{Des bactéries présentes dans l'utérus avant la grossesse}

Depuis maintenant quelques années, grâce à l'amélioration des techniques de microbiologie classique et de biologie moléculaire, des chercheurs ont montré que certains organes, auparavant considérés comme stériles, étaient en fait colonisés par des bactéries même 


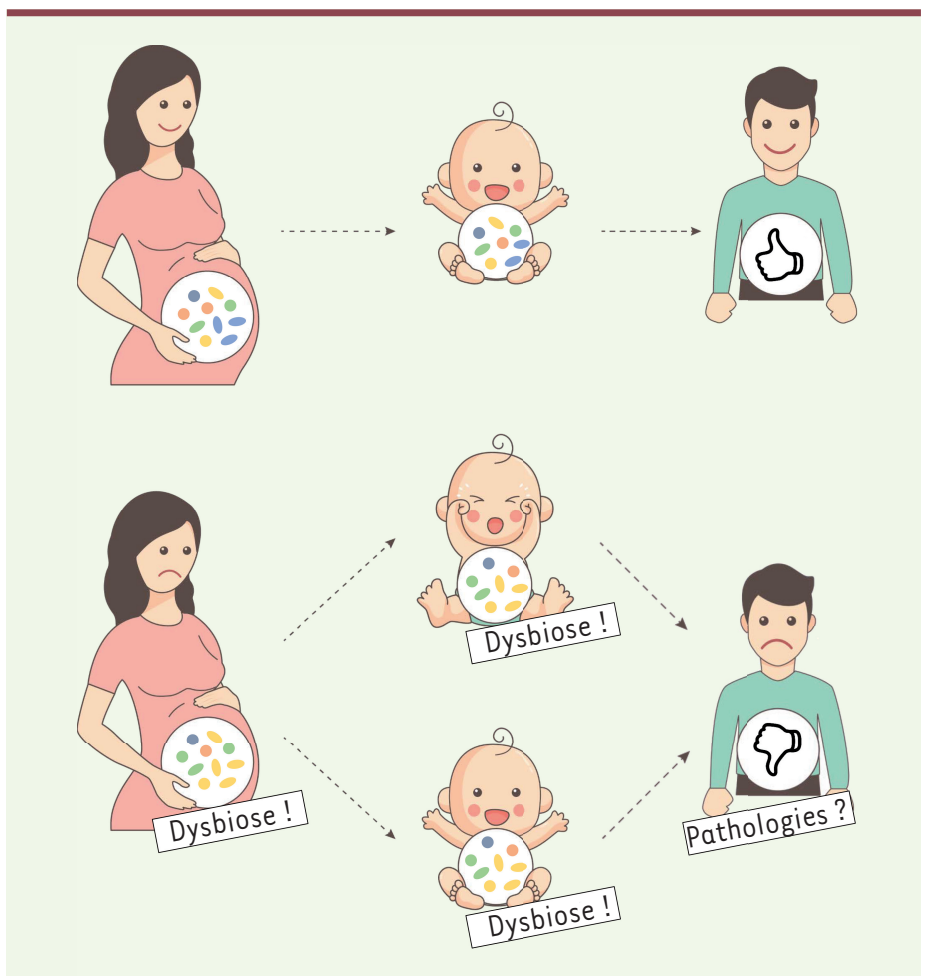

Figure 1. Conséquence de l'existence d'un microbiote in utero. Une dysbiose du microbiote in utero pourrait entraîner une dysbiose chez le nouveau-né. Cette dysbiose pourrait être responsable de pathologies néonatales ou de pathologies à l'âge adulte.

hors contexte infectieux. C'est ainsi qu'un microbiote pulmonaire [46] $(\rightarrow)$

ou encore un microbiote vésical ont été décrits. $(\rightarrow)$ Voir la Synthèse de C.A. Guilloux et al., $m / s n^{\circ} 3$, mars 2018, page 253 L'utérus, lieu de l'implantation de l'embryon et du développement du fœtus, possèderait également son propre microbiote. Alors que la composition du microbiote de la partie inférieure de l'appareil reproductif féminin (cervix, vagin) a été bien étudiée et déterminée, celle de la partie supérieure (ovaires, trompes de Fallope, utérus) n'est encore que peu connue. Ce manque de données s'explique principalement par la difficulté d'accès aux organes. Néanmoins, l'existence d'un microbiote a été montrée par différentes équipes, avec des résultats extrêmement variables. Cette variabilité dans l'identification des populations bactériennes peut s'expliquer par des différences dans les techniques de prélèvement (hystérectomie, aspiration par voie abdominale, aspiration par voie vaginale, biopsie par voie vaginale) mais aussi par les méthodes d'analyse utilisées (biologie moléculaire ou culture bactérienne). Globalement, entre 7 et $100 \%$ des échantillons prélevés par hystérectomie et analysés par culture présentent des bactéries (Tableau I) [15-17]. Les espèces ou genres identifiés sont principalement Bacteroides sp., Gardnerella vaginalis, Streptococcus agalactiae, Staphylococcus epidermidis, Corynebacterium sp. Des lactobacilles et des diphteroides ont également été retrouvés. Les techniques de biologie moléculaire (quelle que soit la technique de prélèvement) révèlent, quant à elles, la présence d'ADN bactérien dans 95 à $100 \%$ des échantillons (Tableau I) [18-20].
Des espèces caractéristiques du microbiote vaginal ont été identifiées avec notamment Lactobacillus crispatus, L. inners, L. jensenni, G. vaginalis, Atopobium vaginae et d'autres espèces telles que Bacteroides xylanisolvens, B. thetaiotamicron, B. fragilis, et des bactéries du genre Prevotella et Sneathia. Malgré leur variabilité, ces données semblent indiquer la présence au niveau de l'utérus, de bactéries qui pourraient jouer un rôle dans l'implantation de l'embryon ou constituer le premier inoculum microbien du futur fœtus. Des recherches sont cependant nécessaires pour caractériser plus précisément ce microbiote et ses fonctions, en particulier lors d'une grossesse.

\section{Des bactéries présentes in utero durant la grossesse}

Baignade en eaux troubles : le liquide amniotique est colonisé

Durant la grossesse, la cavité utérine est remplie de liquide amniotique contenu entre les membranes fœtales dans lequel baigne le fœtus. Il était décrit comme étant stérile et présentant une certaine activité antimicrobienne [21]. Le liquide amniotique peut être prélevé soit au moment de l'accouchement, mais avec une probabilité élevée de contamination par des bactéries exogènes, ou par amniocentèse. Cette technique est cependant invasive et présente un risque pour la grossesse et/ou le fœtus. Elle n'est donc prescrite que dans des cas très précis de suspicion d'infection, de malformation fœtale ou pour détecter des maladies génétiques. L'accessibilité au microbiote présent dans le liquide amniotique reste donc limitée. Dans cette revue, seules les études incluant un groupe de patientes ayant subi une amniocentèse avec des membranes fœtales intactes ont été retenues.

Chez des patientes ne présentant pas de travail prématuré, la présence de bactéries dans le liquide amniotique a pu être évaluée avec cependant des fréquences variables : entre 0 et $7 \%$ des prélèvements par analyse par culture, et entre 0 et $71 \%$ par analyse par PCR (polymerase chain reaction) (Tableau I) [2225]. Les bactéries isolées étaient Ureaplasma urealyticum, S. epidermidis, Fusobacterium nucleatum, Propionibacterium acnes ou certaines espèces du genre Streptococcus ou Corynebacterium. Une étude réalisée par pyroséquençage chez 15 patientes a révélé un microbiote présentant une faible abondance, une faible richesse et une faible diversité bactériennes [26]. Dans cette étude, les protéobactéries étaient les plus représentées avec une majorité d'espèces appartenant aux genres Enterobacter et Escherichial Shigella. Des bactéries du genre Propionibacterium 


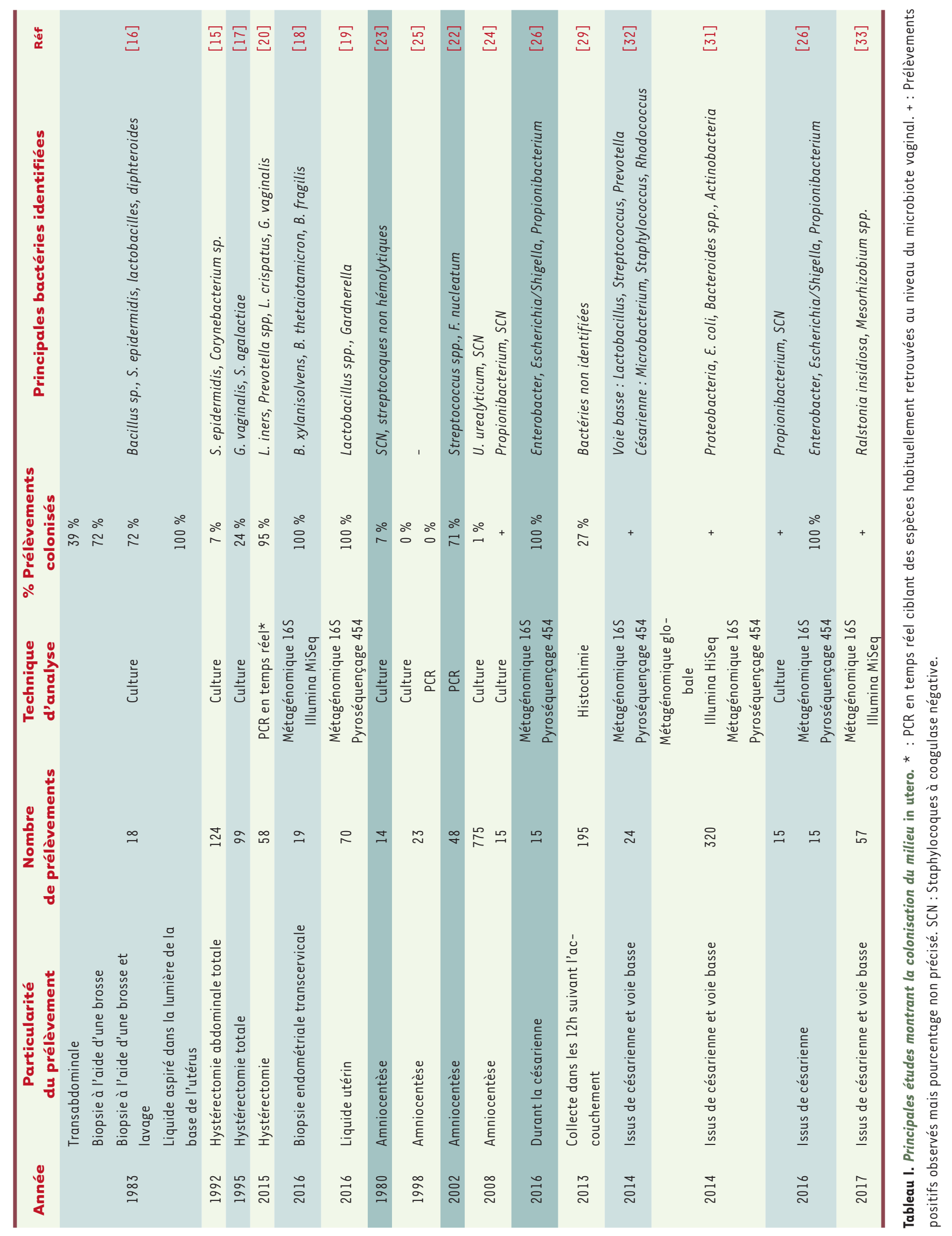




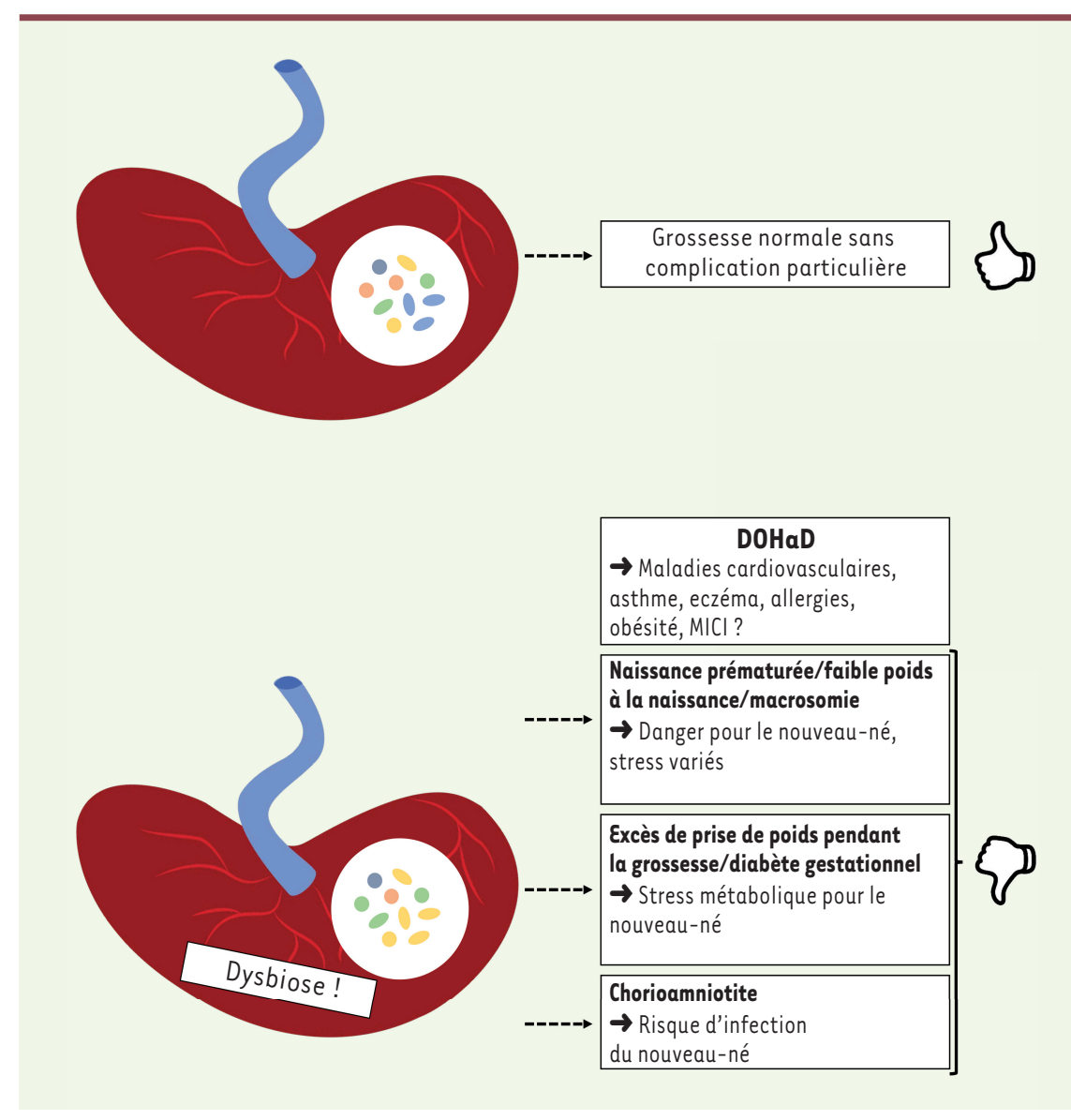

Figure 2. Microbiote placentaire dans la théorie de la DOHaD (developmental origin of health and diseases). Des dysbioses du microbiote placentaire ont été associées à diverses complications de la grossesse pouvant avoir un impact sur le développement du fœtus et/ou sur le nouveau-né. Ces complications peuvent alors être associées au concept de la DOHaD.

bacteria (notamment Escherichia coli) et la présence de Tenericutes [31]. Les autres espèces retrouvées sont, par exemple, Bacteroides spp., Neisseria lactamica, S. epidermidis, P. acnes. Une origine buccale du microbiote placentaire a été proposée dans cette étude, des similitudes ayant été retrouvées avec la composition du microbiote oral décrite dans la littérature. D'autres études ont rapporté la présence d'un microbiote placentaire avec des résultats différents pour sa composition $[26,32,33]$, et suggérant une origine différente comme une origine hématogène (provenant de la translocation bactérienne au niveau de l'épithélium

ont également été retrouvées dans des cultures. Ces microorganismes pourront donc entrer en contact avec le fœtus après qu'il ait dégluti le liquide amniotique.

\section{Un microbiote placentaire au centre d'un échange bactérien entre la mère et le fœtus?}

Le placenta est un organe spécifique et primordial pour le bon déroulement de la grossesse. II est caractérisé par une forte régulation immunitaire en raison de la présence d'antigènes paternels. Son bon fonctionnement est important et une dysfonction placentaire pourrait avoir des conséquences sur la santé future de l'enfant [4]. II y a encore quelques années, seule la recherche de bactéries pathogènes était effectuée dans le placenta en cas de suspicion d'infection maternelle ou néonatale. Au cours de ces études, des bactéries commensales ont cependant été isolées en dehors de tout contexte infectieux [27, 28]. Plus récemment, des bactéries intracellulaires ont été observées, lors de grossesses normales, au niveau des trophoblastes extra-villeux, qui correspondent aux cellules du placenta envahissant l'endomètre maternel [29]. Des bactéries ont également été retrouvées par culture, au niveau du cordon ombilical, suggérant une colonisation possible du fœtus par voie hématogène [30]. Grâce aux techniques de métagénomique, un microbiote placentaire a récemment été caractérisé lors de grossesses normales. Ce microbiote a été qualifié de microbiote spécifique, avec une faible richesse mais une prépondérance en Proteo- intestinal) ou encore une origine ascendante, depuis l'appareil reproductif inférieur. Peu d'études ont été réalisées par culture bactérienne posant la question de la viabilité de ce microbiote placentaire. Ainsi, seuls $P$. acnes et des espèces du genre Staphylococcus ont été retrouvés par culture [26].

Le microbiote placentaire est un microbiote en contact avec le fœtus très précocement. II pourrait ainsi présenter des fonctions physiologiques qui ne sont pas encore élucidées. Des profils particuliers de ce microbiote ont cependant été associés à des complications de la grossesse, comme la naissance prématurée, le diabète gestationnel, la macrosomie fœtale, la chorioamniotite, la pré-éclampsie et le faible poids à la naissance, toutes ces complications pouvant influencer le devenir de l'enfant [31,34-39]. Actuellement, il n'existe néanmoins pas de données sur les effets à long terme d'une dysbiose touchant le microbiote placentaire, celui-ci étant de découverte récente. Il apparaît cependant, que cette dysbiose étant associée à des complications de la grossesse et ces complications pouvant avoir des conséquences sur la santé à long terme, elle pourrait être associée au concept de la DOHaD (Figure 2). 


\section{Microbiote in utero et controverses}

L'ensemble des résultats obtenus jusqu'à présent révèle la faible biomasse que représente le microbiote in utero. Malgré les études montrant son existence, d'autres, contradictoires, et des revues remettent en cause certaines des techniques utilisées pour son identification [40-42].

\section{Fiabilité des techniques de prélèvements}

La comparaison de différentes études révèle des différences de méthodes utilisées pour les prélèvements qui pourraient expliquer la variabilité des résultats obtenus. Ainsi, l'identification des bactéries dans le méconium dépend du délai entre la naissance et son prélèvement [14]. Or, dans certaines études, ce délai n’est pas précisé. On peut donc douter de la représentativité de l'environnement in utero par le méconium analysé. De même, pour les études du microbiote intra-utérin hors grossesse, les techniques utilisées pourraient être à l'origine de contaminations en particulier pour les prélèvements qui nécessitent un passage par le vagin. Les données obtenues sont extrêmement variables; ceci peut s'expliquer par le fait que les échantillons prélevés diffèrent selon les études : fluide utérin ou biopsie utérine en fonction de la technique de prélèvement employée.

Pour le microbiote placentaire, les analyses réalisées par prélèvements par voie basse ne peuvent exclure des contaminations par les microbiotes vaginal et fécal à l'origine de variabilité dans les résultats.

\section{Validité des techniques d'analyses}

La variabilité des résultats peut être reliée à l'hétérogénéité des méthodes d'étude du microbiote. Une étude parue très récemment n'a ainsi pas mis en évidence, par plusieurs techniques de biologie moléculaire (PCR en temps réel ou métagénomique), d'ADN bactérien au sein du placenta [42]. Ces travaux ont aussi montré une probable contamination des kits d'extraction par de l'ADN bactérien... Les études les plus récentes utilisent la biologie moléculaire pour caractériser les microbiotes au détriment de la culture bactérienne. La biologie moléculaire, qui permet d'identifier un plus grand nombre de bactéries, ne permet pas de statuer sur leur viabilité. L'ADN bactérien détecté pourrait s'avérer n'être que des fragments d'ADN provenant de la circulation sanguine, ou de la lyse de cellules immunitaires infectées ou ayant phagocyté les microorganismes. II faut cependant noter que l'ADN, à lui seul, peut avoir un rôle physiologique et stimuler le système immunitaire. Dans le but de comparer l'ensemble de ces données et obtenir des résultats non contestables, il serait important d'harmoniser les techniques de prélèvements et d'analyse de ces microbiotes de faible biomasse.

\section{Conclusion}

L'environnement in utero, contrairement à ce que l'on pensait encore très récemment, semble être colonisé hors contexte infectieux par des bactéries. Un microbiote de faible biomasse a été caractérisé dans différents sites hors et pendant la grossesse et une dysbiose au niveau du microbiote placentaire pourrait être à l'origine de pathologies de la grossesse. Elle pourrait aussi avoir un impact sur la santé future de l'enfant [10]. Son origine reste encore inconnue. Une première hypothèse est une origine hématogène avec des bactéries qui pourraient provenir de différents sites maternels (bouche, intestin), transloquer vers la circulation sanguine et atteindre ainsi le placenta. Une origine ascendante depuis le vagin, ou une origine péritonéale avec le passage des membranes ont également été proposées. Quelle que soit son origine, sa paucité en fait un microbiote difficile à étudier. Cependant, sa caractérisation précise en conditions normales et pathologiques reste indispensable pour comprendre son rôle physiopathologique. La confirmation du rôle de ce microbiote dans le devenir de la grossesse et/ ou la santé de l'enfant ainsi que l'étude des différents paramètres environnementaux qui pourraient l'influencer (terrain génétique, antibiotiques, nutrition, obésité, complications de la grossesse) pourraient ouvrir de nouvelles pistes pour la prise en charge des grossesses à risque ou améliorer la santé future de l'enfant. II pourrait, par exemple, être envisagé de supplémenter la femme enceinte avec des probiotiques, microorganismes vivants qui, ingérés en quantité adéquate, confèrent un bénéfice à l'hôte. L'objectif pourrait être de favoriser ou rétablir un bon équilibre du microbiote in utero. De tels essais cliniques ont été réalisés dans de rares études $[43,44]$. Notons que l'administration de probiotiques chez la femme enceinte a déjà été recommandée par l'organisation mondiale de l'allergie (World Allergy Organization - WAO) dans le cadre de la prévention de l'eczéma chez l'enfant à haut risque atopique [45].

Malgré les controverses soulevées par certaines études, un faisceau de preuves de la présence d'un microbiote in utero commence à exister. Les rôles physiologiques et pathologiques de ce microbiote restent néanmoins à préciser, ce qui représente un défi intéressant dans la prévention de pathologies de la grossesse et de la santé du futur enfant. $\diamond$

\section{SUMMARY}

Microbiota establishment: an in utero colonization decisive for future health?

Some diseases seem to have a developmental origin. Today, the microbiota is recognized as a determinant in health and diseases and one important step is its establishment in the neonate. Some variations in its composition including an imbalance (also called dysbiosis) have been associated to several pathologies. Recent studies suggest a bacterial colonization in the non-pregnant uterus, in the amniotic fluid and in the placenta, which were previously thought sterile. So, during deve- 
lopmental phases, the fetus could have encounter bacteria in utero. These bacteria could contribute to its microbiota establishment before parturition and therefore before the encounter with all microorganisms from vaginal, fecal and cutaneous microbiotas according to the delivery mode. However, studies stating the existence of such in utero microbiota, characterized by a low biomass, are somewhat disputed. $\diamond$

\section{REMERCIEMENTS}

Nous remercions Jessica Richer pour son aide dans la réalisation des figures.

\section{LIENS D'INTÉRÊT}

Les auteurs déclarent n'avoir aucun lien d'intérêt concernant les données publiées dans cet article.

\section{RÉFÉRENCES}

1. Charles MA, Delpierre C, Bréant B. Le concept des origines développementales de la santé : évolution sur trois décennies. Med Sci (Paris) 2016 ; 32 : 15-20.

2. Perera F, Herbstman J. Prenatal environmental exposures, epigenetics, and disease. Reprod Toxicol $2011 ; 31: 363-73$.

3. Drisse MNB. Environnements précoces, origines précoces de la santé et des maladies. Med Sci (Paris) $2016 ; 32: 9-10$.

4. Burton GJ, Fowden AL, Thornburg KL. Placental origins of chronic disease. Physiol Rev 2016 ; 96 : 1509-65.

5. Sender R, Fuchs S, Milo R. Revised estimates for the number of human and bacteria cells in the body. PLoS Biol 2016 ; 14 : e1002533.

6. Hooks KB, O’Malley MA. Dysbiosis and its discontents. mBio 2017 ; 8 : e01492-17.

7. Sekirov I, Russell SL, Antunes LCM, et al. Gut microbiota in health and disease. Physiol Rev 2010 ; $90: 859-904$.

8. Sokol H, Galperine T, Kapel N, et al. Faecal microbiota transplantation in recurrent Clostridium difficile infection: recommendations from the French group of faecal microbiota transplantation. Dig Liver Dis $2016 ; 48: 242-7$.

9. Bakker GJ, Nieuwdorp M. Fecal microbiota transplantation: therapeutic potential for a multitude of diseases beyond Clostridium difficile. Microbiol Spectr 2017 ; 5. doi: 10.1128/microbiolspec. BAD-0008-2017.

10. Tamburini $\mathrm{S}$, Shen $\mathrm{N}, \mathrm{Wu} H \mathrm{HC}$, et al. The microbiome in early life: implications for health outcomes. Nat Med 2016; $22: 713-22$.

11. Indrio F, Martini S, Francavilla R, et al. Epigenetic matters: the link between early nutrition, microbiome, and long-term health development. Front Pediatr $2017 ; 5: 178$.

12. Meropol SB, Edwards A. Development of the infant intestinal microbiome: a bird's eye view of a complex process. Birth Defects Res C Embryo Today 2015 ; 105 : 228-39.

13. Tun HM, Konya T, Takaro TK, et al. Exposure to household furry pets influences the gut microbiota of infant at 3-4 months following various birth scenarios. Microbiome $2017 ; 5: 40$.

14. Jiménez $\varepsilon$, Marín ML, Martín R, et al. Is meconium from healthy newborns actually sterile? Res Microbiol $2008 ; 159: 187-93$.

15. Chimura T, Hirayama T, Morisaki $\mathrm{N}$, et al. Comparisons of the bacterial flora in genital regions at non-pregnancy. Jpn J Antibiot $1992 ; 45: 1065-70$.

16. Duff P, Gibbs RS, Blanco JD, et al. Endometrial culture techniques in puerperal patients. Obstet Gynecol 1983; $61: 217-22$.

17. Møller BR, Kristiansen FV, Thorsen P, et al. Sterility of the uterine cavity. Acta Obstet Gynecol Scand $1995 ; 74: 216-9$.

18. Verstraelen H, Vilchez-Vargas R, Desimpel F, et al. Characterisation of the human uterine microbiome in non-pregnant women through deep sequencing of the V1-2 region of the $16 \mathrm{~S}$ rRNA gene. PeerJ 2016 ; 4 : el602.

19. Moreno I, Codoñer FM, Vilella F, et al. Evidence that the endometrial microbiota has an effect on implantation success or failure. Am J Obstet Gynecol $2016 ; 215: 684-703$.

20. Mitchell CM, Haick A, Nkwopara $\varepsilon$, et al. Colonization of the upper genital tract by vaginal bacterial species in nonpregnant women. Am J Obstet Gynecol $2015 ; 212$ : 611.el-9.

21. Schlievert P, Larsen B, Johnson W, et al. Bacterial growth inhibition by amniotic fluid. III. Demonstration of the variability of bacterial growth inhibition by amniotic fluid with a new platecount technique. Am J Obstet Gynecol 1975; 122 : 809-19.

22. Bearfield C, Davenport $E S$, Sivapathasundaram V, et al. Possible association between amniotic fluid micro-organism infection and microflora in the mouth. BJOG $2002 ; 109: 527-33$.

23. Miller Jr JM, Pupkin MJ, Hill GB. Bacterial colonization of amniotic fluid from intact fetal membranes. Am J Obstet Gynecol $1980 ; 136: 796-804$.

24. Seong HS, Lee SE, Kang JH, et al. The frequency of microbial invasion of the amniotic cavity and histologic chorioamnionitis in women at term with intact membranes in the presence or absence of labor. Am J Obstet Gynecol 2008 ; 199 : 375.el-5.
25. Oyarzún $\varepsilon$, Yamamoto M, Katoa $S$, et al. Specific detection of 16 microorganisms in amniotic fluid by polymerase chain reaction and its correlation with preterm delivery occurrence. Am J Obstet Gynecol 1998; 179 : 1115-9.

26. Collado MC, Rautava S, Aakko J, et al. Human gut colonisation may be initiated in utero by distinct microbial communities in the placenta and amniotic fluid. Sci Rep $2016 ; 6: 23129$.

27. Pettker CM, Buhimschi IA, Magloire LK, et al. Value of placental microbial evaluation in diagnosing intra-amniotic infection. Obstet Gynecol 2007 ; 109 : 739-49.

28. Jones $H E$, Harris KA, Azizia $M$, et al. Differing prevalence and diversity of bacterial species in fetal membranes from very preterm and term labor. PloS One 2009; 4 : e8205.

29. Stout MJ, Conlon B, Landeau M, et al. Identification of intracellular bacteria in the basal plate of the human placenta in term and preterm gestations. Am J Obstet Gynecol $2013 ; 208$ : 226.el-7.

30. Jiménez $\varepsilon$, Fernández L, Marín ML, et al. Isolation of commensal bacteria from umbilical cord blood of healthy neonates born by cesarean section. Curr Microbiol $2005 ; 51: 270-4$.

31. Aagaard K, Ma J, Antony KM, et al. The placenta harbors a unique microbiome. Sci Transl Med $2014 ; 6$ : 237 ra65.

32. Doyle RM, Alber DG, Jones HE, et al. Term and preterm labour are associated with distinct microbial community structures in placental membranes which are independent of mode of delivery. Placenta 2014 ; 35 : 1099-101.

33. Parnell LA, Briggs CM, Cao B, et al. Microbial communities in placentas from term normal pregnancy exhibit spatially variable profiles. Sci Rep 2017 ; 7 : 11200.

34. Prince AL, Ma J, Kannan PS, et al. The placental microbiome is altered among subjects with spontaneous preterm birth with and without chorioamnionitis. Am J Obstet Gynecol.2016 ; 214 : 627.el-16.

35. Barak $S$, Oettinger-Barak 0 , Machtei $\varepsilon \varepsilon$, et al. Evidence of periopathogenic microorganisms in placentas of women with preeclampsia. J Periodontol $2007 ; 78: 670-6$.

36. Zheng J, Xiao X, Zhang $Q$, et al. The placental microbiome varies in association with low birth weight in full-term neonates. Nutrients $2015 ; 7$ : 6924-37.

37. Zheng J, Xiao X, Zhang $Q$, et al. Correlation of placental microbiota with fetal macrosomia and clinical characteristics in mothers and newborns. Oncotarget $2017 ; 8: 82314-25$.

38. Zheng J, Xiao X, Zhang $Q$, et al. The placental microbiota is altered among subjects with gestational diabetes mellitus: A pilot study. Front Physiol $2017 ; 8: 675$.

39. Bassols J, Serino M, Carreras-Badosa G, et al. Gestational diabetes is associated with changes in placental microbiota and microbiome. Pediatr Res $2016 ; 80: 777-84$.

40. Perez-Muñoz ME, Arrieta MC, Ramer-Tait AE, et al. A critical assessment of the sterile womb and in utero colonization hypotheses: implications for research on the pioneer infant microbiome. Microbiome $2017 ; 5: 48$.

41. Kliman HJ. Comment on the placenta harbors a unique microbiome. Sci Transl Med $2014 ; 6$ : 254 le4.

42. Lauder AP, Roche AM, Sherrill-Mix S, et al. Comparison of placenta samples with contamination controls does not provide evidence for a distinct placenta microbiota. Microbiome 2016; $4: 29$.

43. Rautava S, Collado MC, Salminen $S$, et al. Probiotics modulate host-microbe interaction in the placenta and fetal gut: a randomized, double-blind, placebo-controlled trial. Neonatology 2012 ; 102 : 178-84.

44. Kavak SB, Kavak $\varepsilon$, Ilhan $R$, et al. The efficacy of ampicillin and Lactobacillus casei rhamnosus in the active management of preterm premature rupture of membranes remote from term. Drug Des Devel Ther $2014 ; 8: 1169-73$.

45. Fiocchi A, Pawankar R, Cuello-Garcia C, et al. World allergy organizationmcmaster university guidelines for allergic disease prevention (GLAD-P): probiotics. World Allergy Organ J $2015 ; 8: 4$.

46. Guilloux CA, Lamoureux C, Héry-Arnaud G. Les bactéries anaérobies, ces inconnues du microbiote pulmonaire. Med Sci (Paris) 2018 ; 34 : 253-60.

47. Junien C (coordination). Origine développementale de la santé et des maladies (DOHaD) : environnement et épigénétique. Med Sci (Paris) 2016 ; $32: 3-130$. 